Revue musicale OICRM

\title{
Le Verfügbar aux Enfers
} Études et mises en scène contemporaines

\section{Cécile Quesney}

Volume 3, numéro 2, 2016

Mémoire musicale et résistance. Autour du Verfügbar aux Enfers de Germaine Tillion

URI : https://id.erudit.org/iderudit/1060108ar

DOI : https://doi.org/10.7202/1060108ar

Aller au sommaire du numéro

\section{Éditeur(s)}

OICRM

\section{ISSN}

2368-7061 (numérique)

Découvrir la revue

\section{Citer cet article}

Quesney, C. (2016). Le Verfügbar aux Enfers : études et mises en scène contemporaines. Revue musicale OICRM, 3(2), 78-98.

https://doi.org/10.7202/1060108ar
Résumé de l'article

Depuis la publication du Verfügbar aux Enfers aux éditions de La Martinière en 2005, le texte de Germaine Tillion a fait l'objet de brèves études et a donné lieu à de nombreuses productions musico-théâtrales, dont la première et la plus célèbre est celle réalisée au Théâtre du Châtelet à Paris en juin 2007. À partir d'une analyse de quelques productions récentes et des textes scientifiques traitant de l'opérette-revue, cet article rend compte des différentes interprétations du Verfügbar aux Enfers proposées par les chercheurs, metteurs en scène et adaptateurs du texte de Tillion. Il apparaît notamment que la démarche du Châtelet, caractérisée par la création d'une partition ainsi que par une approche pédagogique et mémorielle, est souvent reprise pour de nouveaux spectacles. Sont également examinées quelques mises en scène davantage centrées sur la forme et le sens du texte. 


\title{
Le Verfügbar aux Enfers. Études et mises en scène contemporaines ${ }^{1}$

\author{
Cécile Quesney
}

\begin{abstract}
Résumé
Depuis la publication du Verfügbar aux Enfers aux éditions de La Martinière en 2005, le texte de Germaine Tillion a fait l'objet de brèves études et a donné lieu à de nombreuses productions musico-théâtrales, dont la première et la plus célèbre est celle réalisée au Théâtre du Châtelet à Paris en juin 2007. À partir d'une analyse de quelques productions récentes et des textes scientifiques traitant de l'opérette-revue, cet article rend compte des différentes interprétations du Verfügbar aux Enfers proposées par les chercheurs, metteurs en scène et adaptateurs du texte de Tillion. Il apparaît notamment que la démarche du Châtelet, caractérisée par la création d'une partition ainsi que par une approche pédagogique et mémorielle, est souvent reprise pour de nouveaux spectacles. Sont également examinées quelques mises en scène davantage centrées sur la forme et le sens du texte.
\end{abstract}

Mots clés : interprétation ; Le Verfügbar aux Enfers ; mise en scène ; résistance ; Germaine Tillion.

\begin{abstract}
Since the publication of Le Verfügbar aux Enfers by La Martinière in 2005, Germaine Tillion's text has given rise to a number of short studies as well as to several music and theatrical productions. The first and most famous of these was at Paris' Théâtre du Châtelet in June 2007. Based on an analysis of recent productions and scholarly articles on Tillion's opérette-revue, this paper discusses the various interpretations of Le Verfügbar aux Enfers that have been suggested by researchers, directors and adapters of Tillion's text. It appears that the approach used by the Théâtre du Châtelet, one characterized both by the production of a musical score and a pedagogical and memorial concern, has been frequently reused in subsequent productions. This article also considers some theatrical productions that focus more closely on the form and meaning of the text.
\end{abstract}

Keywords: interpretation; Le Verfügbar aux Enfers; production; resistance; Germaine Tillion.

$1 \quad$ Nous adressons nos plus vifs remerciements à l'Association Germaine Tillion ainsi qu'à Nelly Forget, Jacky Kerneur, Anise Postel-Vinay, Roselyne Sarazin et Danielle Stéfan qui nous ont transmis des informations et des documents essentiels sur l'opérette-revue et ses mises en scène. Nous remercions également Marie-Hélène Benoit-Otis, Esteban Buch et Philippe Despoix pour leurs bons conseils et leurs relectures attentives. 
L'une des grandes singularités de l'opérette-revue de Germaine Tillion tient au fait qu'elle n'a pas été conçue pour être représentée. Travail d'écriture-performance collective, composé par mais surtout pour les prisonnières elles-mêmes sous la direction de Tillion, Le Verfügbar aux Enfers a été écrit dans le but d'être lu, dit, chantonné et murmuré de façon parcellaire et dans le plus grand secret - il était en effet tout à fait impensable d'interpréter une telle ouvre dans son intégralité et a fortiori de la représenter au sein du camp. Tillion et ses camarades n'ont pu que lire et chanter des extraits de l'opérette au fur et à mesure de son élaboration, au block ou dans les colonnes de travail, et faire circuler le manuscrit en cachette afin que d'autres prisonnières puissent le consulter (Toulouse-Lautrec 1981, p. 273).

Lorsqu'elle est libérée par la Croix-Rouge suédoise le 23 avril 1945 avec plusieurs centaines de détenues, Tillion fait sortir clandestinement le manuscrit du Verfügbar avec d'autres documents contenant des informations de premier ordre sur le fonctionnement du camp ${ }^{2}$. Mais ce n'est que bien des années après la guerre que Le Verfügbar aux Enfers sort véritablement de l'ombre. Tillion en cite d'abord des extraits dans son troisième Ravensbrück paru en 1988 (Tillion 1988), et le publie finalement comme un opus à part entière en 2005 (Tillion 2005) ${ }^{3}$. Cette publication marque les débuts de la seconde vie de l'opérette-revue ; rendu accessible, son texte devient par cette occasion une source et un objet d'étude pour les chercheurs, mais aussi une œuvre destinée à la scène. Or depuis la sortie du livre l'investissement du monde du spectacle à son sujet paraît plus important que celui de la recherche, les études menées étant généralement peu développées. Comment expliquer un tel décalage?

La première raison semble évidente : le texte de Tillion a fait l'objet d'une quinzaine de mises en scène parce qu'il s'agit bien d'une "opérette-revue », comme l'indique d'ailleurs son sous-titre. Même si ses auteures n'ont pas songé à le porter à la scène au moment où elles l'ont conçu, le texte est bel est bien pensé comme une ouvre de théâtre ; rien de plus naturel, dès lors, que le monde du spectacle s'en soit emparé. Inversement, la nature complexe et transdisciplinaire de cet objet théâtral, qui se situe au croisement de différents domaines artistiques et des sciences humaines (il s'agit à la fois d'un texte de théâtre avec danse et musique, d'un témoignage et d'un document ethnographique) explique probablement qu'il ait suscité peu de travaux scientifiques d'envergure pour le moment. La dimension restreinte des études consacrées au Verfügbar aux Enfers tient aussi, bien sûr, au caractère récent de sa publication. À cet égard, l'entrée au Panthéon de Germaine Tillion en $2015^{4}$ contribuera sans doute à mieux faire connaître cette œuvre singulière, notamment dans le milieu académique.

2 C'est Jacqueline d'Alincourt qui sort le manuscrit du camp (« Avant-propos » de Tzvetan Todorov, Tillion 2005, p. 3).

3 Sur la sauvegarde et la publication du Verfügbar aux Enfers, voir également l'article de Marie-Hélène Benoit-Otis et Philippe Despoix dans le présent numéro.

$4 \quad$ Notons que lors de la cérémonie du Panthéon du 27 mai 2015, le Président de la République François Hollande a mentionné dans son discours l'existence de l'opérette-revue conçue, dit-il, pour « pouvoir défier le mal par le rire". 
Pour l'heure, trois tendances dans l'interprétation - dans tous les sens du terme de l'opérette-revue peuvent être mises en évidence : du côté des études scientifiques, Le Verfügbar aux Enfers est surtout considéré comme un document historique mais ne fait pas encore l'objet d'analyses musico-littéraires poussées - l'un des objectifs du groupe de recherche basé à l'Université de Montréal consiste d'ailleurs à pallier ce manque ${ }^{5}$; pour ce qui est des productions musicales et théâtrales, on peut d'abord dégager une majorité de mises en scènes qui, à l'instar de la production du Châtelet en 2007, sont fondées sur une lecture avant tout musicale de l'œuvre tout en accordant une large place à ses enjeux mémoriels et pédagogiques ; et enfin, on peut distinguer quelques productions davantage centrées sur le texte de Tillion et qui s'éloignent à plusieurs égards de l'exemple du Châtelet.

\section{LE VERFÜGBAR AUX ENFERS COMME SOURCE ET COMME OBJET D'ÉTUDE}

\section{L'édition du texte}

Révélant au grand public un texte resté pendant plusieurs décennies dans des archives privées, la première édition du manuscrit de Tillion paraît aux éditions de La Martinière en 2005. Chaque page transcrite (page de gauche) est accompagnée de la reproduction de l'original en page de droite, et une copie complète du manuscrit en facsimilé est proposée sous la forme d'un petit livret d'accompagnement.

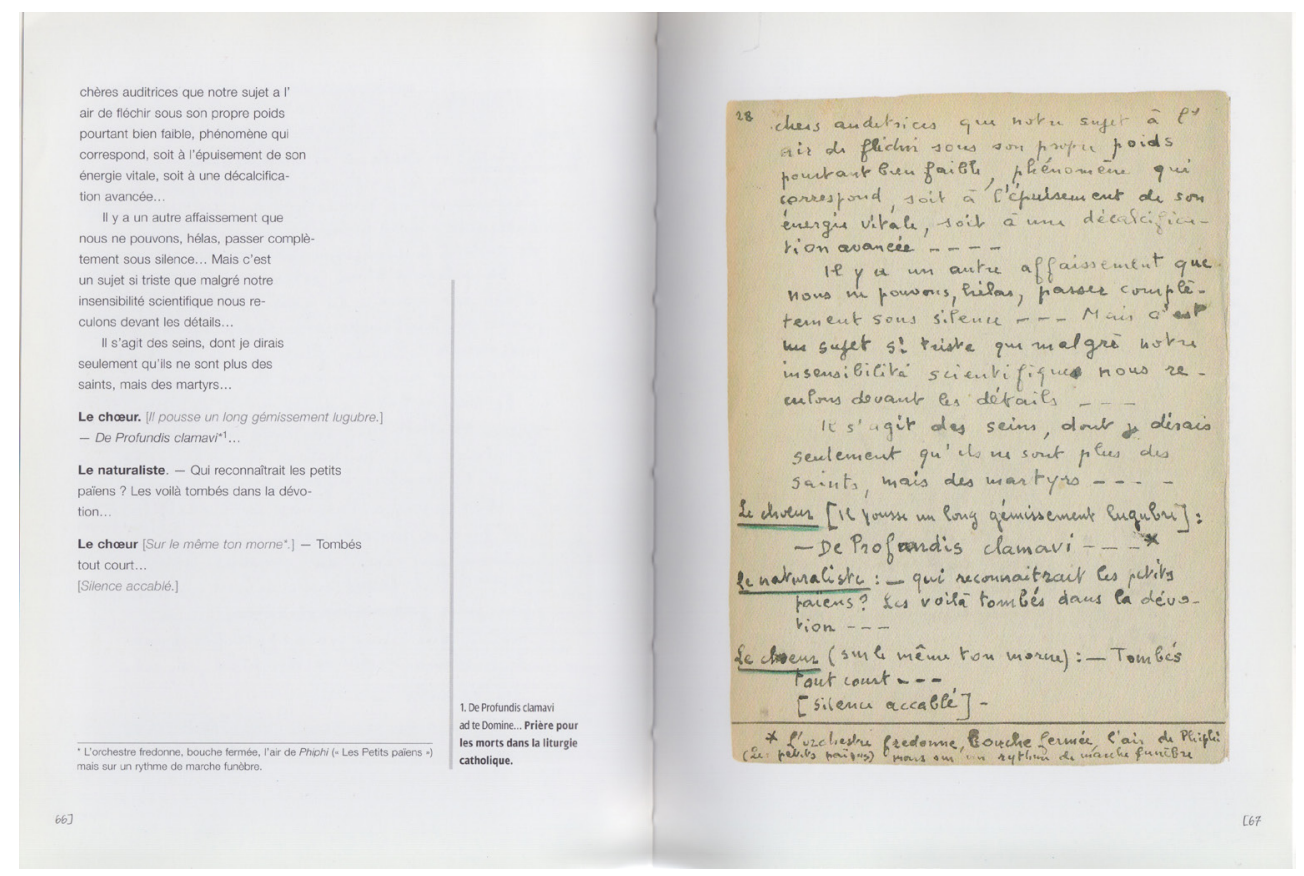

Figure 1 : Germaine Tillion, extrait du Verfügbar aux Enfers (Tillion 2005, p. 66-67).

5 Voir à ce sujet l'introduction du présent numéro, ainsi que l'article de Marie-Hélène Benoit-Otis et Philippe Despoix. 
Ce choix d'offrir une copie de l'objet s'avère particulièrement pertinent et précieux pour le lecteur qui peut ainsi mieux cerner la nature singulière de ce manuscrit musico-théâtral sans portées, petit cahier de fortune constitué de feuillets volés au camp par une camarade tchèque ${ }^{6}$ de Kouri (nom de prisonnière de Tillion) et sur lesquels l'ethnologue a fixé de son écriture précise et serrée le texte et les sources musicales ("sur l'air de ») de son opérette-revue. Le livret facsimilé permet en outre de reproduire dans son contexte le dessin de France Audoul qui orne le " Prologue » de la pièce, ainsi que les différentes couleurs d'encre utilisées par Tillion.

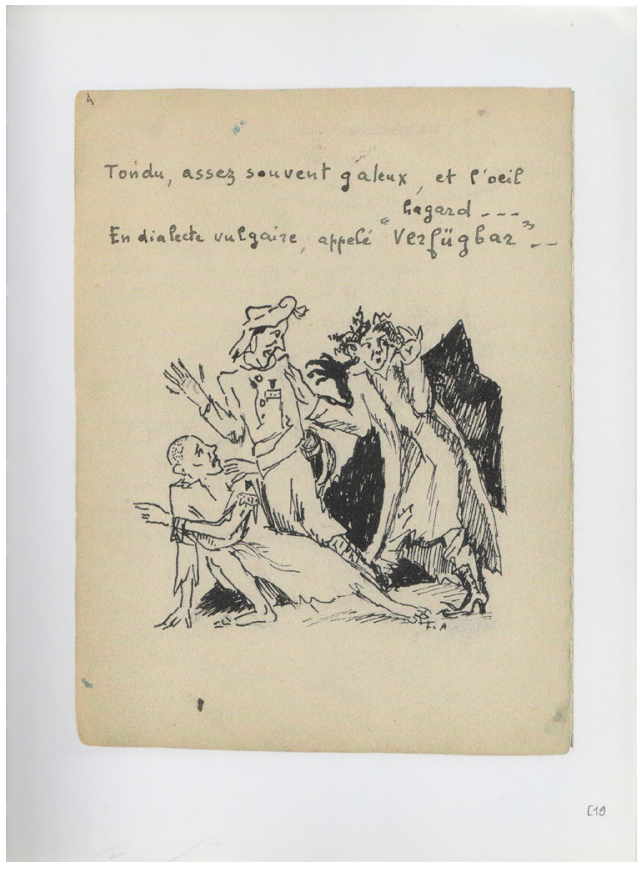

Figure 2 : Germaine Tillion, extrait du Verfügbar aux Enfers (Tillion 2005, p. 19). Dessin de France Audoul en page 4 du manuscrit.

Dans la partie " livre », le texte est annoté par Anise Postel-Vinay, camarade de Tillion à Ravensbrück, tandis que les sources musicales sont établies par Nelly Forget, membre fondateur de l'Association Germaine Tillion et proche de Tillion depuis leur engagement respectif en Algérie (ibid., p. 220-223). L'avant-propos de Tzvetan Todorov met l'accent sur le caractère singulier de cette œuvre issue de l'univers concentrationnaire, où objectivité et autodérision sont perpétuellement mêlées. Enfin, l'introduction de l'historienne Claire Andrieu permet de contextualiser et de saisir les principales caractéristiques de ce texte conçu pour " faire rire, rire de soi et transmettre l'information " (ibid., p. 6). Cette première édition a connu un certain succès commercial, avec près de 4000 exemplaires vendus depuis sa parution. L'édition de poche qui lui a succédé a paru chez Points Seuil en 2007 et a elle été vendue à près de 9000 exemplaires ${ }^{7}$.

6 Germaine Tillion par elle-même, film documentaire réalisé par Alain Moreau et l'Association Germaine Tillion, 2015, 29:20-29:39.

7 Chiffres de vente transmis par l'éditeur. 
Les premières études : approches historique et esthétique

Quelques sources musicales demeurées inconnues jusqu'en 2005 ont été identifiées depuis la parution du livre. La publication a en effet entraîné plusieurs recherches musicologiques et historiques autour du Verfügbar aux Enfers. Outre le travail d'identification des sources musicales d'abord mené par Forget, on peut distinguer d'une part un travail de recherche similaire mais motivé et donc orienté par un projet de performance des passages musicaux, tel celui réalisé par la musicologue Lucie Kayas et le compositeur Christophe Maudot en vue de la création de l'œuvre au Théâtre du Châtelet à Paris en 2007 (voir infra) ; on peut d'autre part identifier des recherches de nature historique ou esthétique, dans lesquelles Le Verfügbar aux Enfers constitue la source et/ou l'objet du chercheur. Ces dernières études totalement ou partiellement consacrées à l'opérette-revue sont encore assez peu nombreuses. La première semble être l'article de l'historien Donald Reid paru en 2007 dans une revue d'histoire américaine (Reid 2007a). S'appuyant principalement sur le texte de l'opérette et dans une moindre mesure sur des témoignages publiés de résistants déportés et des ouvrages sur l'histoire et la mémoire des camps, Reid s'intéresse à l'histoire des prisonnières "Verfügbar " et à l'opérette de Tillion en tant que manifestations de la résistance des Françaises à Ravensbrück. Il insiste particulièrement sur la démarche de ces détenues qui choisissent délibérément d'appartenir à cette catégorie des "Verfügbar" (" disponibles $\left.{ }^{8} »\right)$ - c'est-à-dire à la classe la plus misérable et la plus vulnérable de la population des prisonnières - afin de ne pas participer à l'effort de guerre allemand, ce refus du travail impliquant de se cacher en permanence. Reid met ainsi en évidence l'esprit de résistance inhérent au Verfügbar, qui refuse par principe de se soumettre et de travailler pour l'ennemi. Le Verfügbar refuse également de subir l'entreprise nazie de déshumanisation des prisonnières ; 1'opérette-revue de Tillion, tout comme l'écriture et la circulation de poèmes parmi les détenues, participe de cette autre forme de résistance. Reid insiste par ailleurs sur le personnage du naturaliste, qui dans l'opérette joue le rôle d'un ethnographe mais raciste et incapable d'humanité, ainsi que sur l'humour et l'autodérision du Verfügbar aux Enfers. L'auteur retrace enfin l'histoire singulière du texte de Tillion, inséparable de celle de Ravensbrück et de sa mémoire. On le voit, l'objectif de Reid n'est pas d'offrir une analyse détaillée (qu'elle soit historique, dramaturgique, littéraire ou même musicale) du texte ; ce dernier est avant tout considéré comme une trace et un témoignage de la résistance des déportées françaises à Ravensbrück.

Après cet article pionnier - que Reid reprend dans un livre sur la mémoire de la Résistance française paru la même année (Reid 2007b, p. 96-105) - signalons l'étude d'Andrea Loselle (2010) centrée sur les chansons de camp et les pièces en musique de Tillion et Charlotte Salomon créées en déportation (pour Tillion) et en exil (pour Salomon). Dans cet article, Loselle part du constat que l'on connaît mal les œuvres

8 Pour une définition des prisonnières "Verfügbar », voir l'article de Marie-Hélène Benoit-Otis et Philippe Despoix dans le présent numéro. 
théâtro-musicales clandestines issues des camps. Elle remarque que beaucoup d'entre elles sont empreintes d'humour noir et ont une grande valeur documentaire en ce qu'elles représentent directement la réalité des camps. Ses deux principaux exemples, Le Verfügbar aux Enfers de Tillion et Leben? oder Theater? de Charlotte Salomon, ont selon elle plusieurs points communs (relatifs au genre ${ }^{9}$, à l'intertextualité musicale et à la performance intérieure) en dépit du contexte très différent de leur création. Elle souligne notamment que la pièce de Tillion contient des moments musicaux et théâtraux dérivant directement des actions accomplies par les prisonnières du camp et que certains passages témoignent d'une forme d'intériorité exprimée par le biais de la musique et d'une écoute intérieure de celle-ci (on chante pour soi ; le chanteur est aussi l'auditeur et vice versa). Loselle s'intéresse ensuite à Leben? oder Theater?, une œuvre autobiographique complexe, sorte de pièce de théâtre musicale et graphique constituée de près de 800 gouaches accompagnées de textes et de citations littéraires et musicales que Salomon réalise entre 1940 et 1942 dans le sud-est de la France après son internement au camp de Gurs (Voolen 2005, Salomon 2015). La jeune femme, juive allemande exilée en France, livre dans ce "Singespiel » le récit de son enfance, de sa jeunesse et de la montée du nazisme. Les références musicales qui truffent le texte sont des citations d'airs connus issus de divers répertoires qui, comme l'indique Salomon dans l'introduction, lui sont venus à l'esprit et qu'elle a fredonnés au moment de la réalisation des gouaches. Loselle n'entre pas dans une analyse détaillée de la pièce de Salomon, déjà étudiée par ailleurs (Steinberg et Bohm-Duchen 2006), mais souligne que les images visuelles et sonores associées au sens tout comme les caractéristiques performatives de cette œuvre (un "théâtre de l'intérieur ", sans spectateur) la rapprochent singulièrement de celle de Tillion.

Les publications récentes : force du rire et théâtre de la résistance

À la suite de l'étude d'orientation esthétique et comparative de Loselle, quelques publications consacrées à des sujets plus larges proposent de brèves analyses de l'opérette-revue (Giner 2011, p. 70-72, Losco-Lena 2011, p. 165-168, Gilzmer 2013, p. 154-156). Mentionnons également l'article de Sylvie Brodziak paru en 2013 et consacré aux "rires de femmes " dans Le Verfügbar aux Enfers. Dans cette étude d'orientation littéraire, l'auteure montre d'abord que la publication tardive de l'opérette-revue s'inscrit dans le récent « processus de détabouisation du comique dans la connaissance de la Catastrophe »(Brodziak 2013, p. 137). Elle met ensuite en évidence les spécificités de l'œuvre : l'autodérision, le caractère collectif de sa genèse et le fait qu'elle témoigne de la grande solidarité des Françaises de Ravensbrück. L'auteure s'intéresse enfin au rire comme expression du corps, mécanisme de survie " permettant de ranimer ce corps que l'on ne reconnaît plus » (ibid., p. 148). Ainsi distingue-t-elle deux types de rires : le " rire de soi » des déportées, et le rire des femmes - « rire genré » qui se moque des corps délabrés.

9 Selon Loselle, Le Verfügbar aux Enfers, et plus généralement la musique créée en déportation, réinvente la " pièce chantée " ("song play») que l'on peut observer à partir du XVII" siècle et selon diverses modalités en Angleterre, en France et en Allemagne 
Signalons également le catalogue de l'exposition "Les armes de l'esprit. Germaine Tillion 1939-1954 » présentée à Besançon à l'automne 2015 et dans lequel figurent trois articles portant de près ou de loin sur 1'opérette-revue (Musée de la Résistance et de la Déportation de Besançon 2015) ${ }^{10}$. Il s'agit d'abord du texte de Claire Andrieu, "Le Verfügbar aux Enfers : une lecture historique » (Andrieu 2015), qui est en fait une réédition de son "Introduction " à l'édition de La Martinière (voir supra). Le catalogue contient également un article de Christophe Maudot abordant la riche culture musicale de Tillion (dont l'opérette-revue constitue un témoignage éloquent) et proposant une enquête sur sa carrière de spectatrice à Paris durant ses années de formation (Maudot 2015). En partant de ses agendas de l'époque et des programmes des salles fréquentées par la jeune femme, l'auteur montre le grand appétit de Tillion pour les concerts et spectacles, et en particulier pour Wagner, Debussy, Honegger, ainsi que pour la musique légère et le divertissement. Enfin, un texte d'Emmanuel Font sur « le rire de Germaine Tillion face à l'expérience concentrationnaire » met en évidence le caractère salutaire du rire et de l'écriture théâtrale pour les déportées (Font 2015). L'auteur s'intéresse également à l'histoire du manuscrit de l'opérette-revue, celle d'un " rire enfoui » (ibid., p. 92) ressurgissant soixante ans après les événements. Il s'interroge ultimement sur la façon de jouer cette " œuvre amusante sur un sujet grave " (ibid., p. 93), évoquant brièvement les divers choix opérés par quelques metteurs en scène contemporains ainsi que le phénomène d'actualisation de la mémoire induit par ces re-présentations de la déportation.

Claire Audhuy, chercheuse en études théâtrales, signe quant à elle les travaux récents les plus substantiels. Dans sa thèse sur le théâtre dans les camps nazis, elle consacre plusieurs dizaines de pages au Verfügbar aux Enfers (Audhuy 2013, p. 187-214, 395-409, 410-425) ; 1'opérette-revue est également 1'objet principal d'un article qu'elle publie l'année suivante dans une revue théâtrale (Audhuy 2014). Dans les deux cas, Audhuy propose une analyse de certains passages de l'œuvre tout en replaçant cette dernière dans son contexte de création. Dans sa thèse, elle détaille les différents types de transgression dont témoigne l'écriture du Verfügbar aux Enfers et parle à son sujet de "résistance théâtrale » (Audhuy 2013, p. 209) et d'une forme de " théâtre documentaire et ethnologique » (ibid., p. 207). Elle s'intéresse plus particulièrement au processus de détournement et de réécriture des sources musicales, qu'elle considère comme autant de commentaires au texte de Tillion, lequel vise notamment à informer ses camarades de la réalité du camp ${ }^{11}$. L'opérette est donc à la fois un vecteur d'informations sur le fonctionnement du camp (et donc un document historique), une œuvre de résistance et une performance musico-théâtrale qui permet aux détenues de s'évader par le rire et l'ironie.

Si les travaux d'Audhuy apportent plusieurs éclairages historiques et littéraires sur l'œuvre, on peut regretter qu'ils n'offrent pas d'analyse fouillée de la pièce et peu de

10 Voir le compte rendu de cet ouvrage signé par Catherine Harrison-Boisvert dans le présent numéro.

11 En faisant comprendre à ses compagnes le fonctionnement du camp, Tillion leur permet de mieux affronter leur condition de détenues. 
points de comparaison avec d'autres œuvres similaires issues des camps. Cette thèse et cet article n'en constituent pas moins les premiers travaux d'envergure sur l'œuvre elle-même, fournissant ainsi une très bonne base de travail pour des études à venir. La thèse s'appuie d'ailleurs sur plusieurs entretiens avec des témoins - rassemblés dans le volume d'annexes - dont certains apportent un éclairage inédit et tout à fait passionnant sur Le Verfügbar aux Enfers. Les entretiens réalisés en 2008 et 2009 avec d'anciennes camarades de block de Tillion, Anise Postel-Vinay et Marie-José Chombart de Lauwe (Audhuy 2013, Annexes, p. 400-422), constituent en effet une précieuse source d'informations sur le contexte de création de l'opérette-revue (en particulier sur les rapports de solidarité et de fraternité tissés par les prisonnières entourant Tillion) et sur sa diffusion au sein du camp. Le témoignage de Chombart de Lauwe met l'accent sur la technique de décentrement à l'œuvre dans Le Verfügbar aux Enfers, une posture qui a permis aux prisonnières de rire d'elles-mêmes, de prendre de la distance face à leur situation et donc de mieux supporter l'insupportable. Le témoignage de Postel-Vinay révèle par ailleurs que Tillion a très probablement opté pour un genre gai et populaire afin de remonter le moral des troupes, mais aussi pour permettre au plus grand nombre de " mettre la main à la pâte, puisque c'était des chansons que tout le monde savait » (ibid., p. 406). Cet entretien apporte également plusieurs éléments de réflexion sur l'adaptation scénique proposée au Châtelet en 2007. Postel-Vinay a en effet suivi de près la préparation de la production parisienne, dont elle se dit très satisfaite ${ }^{12}$.

\section{De PARIS À MARSEILle : REgARDS SUR QUELQUeS PRODUCTIONS FRANÇAISES DE L'OPÉRETTE-REVUE}

\section{La production du Châtelet et ses avatars}

Le projet de mettre en scène pour la première fois Le Verfügbar aux Enfers au Théâtre du Châtelet n'est pas allé de soi, et sa réalisation n'a été décidée qu'après une réflexion menée en concertation avec l'Association Germaine Tillion. Il s'agissait notamment de ne pas «basculer dans le voyeurisme, ni dans la leçon de morale ${ }^{13}$ » et de proposer une mise en scène fidèle et sobre (confiée à Bérénice Collet), moyennant pourtant une adaptation du texte jugée nécessaire du point de vue théâtral. Un document émanant des services du Châtelet explique certains choix d'adaptation et d'interprétation :

La multiplicité des personnages, la spécificité du langage, nous ont conduits à proposer une adaptation qui rende le texte plus théâtral. Ainsi le nombre de personnages a-t-il été réduit. Seront conservés : le Naturaliste (acteur), 6 actrices/chanteuses condensant l'ensemble des personnages féminins (Nénette, Marguerite, Havas, Marmotte, Lulu de Colmar, Lulu de Belleville),

12 Postel-Vinay a elle-même prononcé une courte introduction au spectacle lors des représentations, une mise en contexte que les instigateurs du projet ont sans doute jugée nécessaire à la bonne compréhension de l'œuvre.

13 Document dactylographié sans référence émanant des services du Châtelet, Boîte "Verfügbar ", Chemise « Autour du Verfügbar aux Enfers (2005-2006) », Fonds Tillion, BnF. 
chœur et ballet.

Le chœur sera assuré par deux classes de collégiens issus du XIII ${ }^{\mathrm{e}}$ arrondissement de Paris, qui, soutenus par la Maîtrise de Paris, travailleront en ateliers dès le mois de janvier. Le ballet sera constitué de jeunes danseurs issus des Conservatoires d'arrondissement de Paris ou du Conservatoire National de Région (CNR).

L'arrangement musical pour dix instruments a été confié à un jeune compositeur, Christophe Maudot, prix Claude Arrieu de la SACEM et professeur de composition au CNR de Lyon ${ }^{14}$.

Le choix de proposer un arrangement instrumental, une décision qui semble s'être imposée naturellement, n'est pas expliqué ici. Il s'agit pourtant d'un important parti pris, puisqu'il implique d'adjoindre aux sections chantées un accompagnement instrumental qui n'a pas du tout été pensé par les auteures - le manuscrit ne contenant aucune annotation musicale ou indication d'accompagnement. Le fait que l'arrangement soit conçu pour des voix lyriques - un choix qui privilégie le répertoire classique au détriment des sources populaires ${ }^{15}$ et témoigne de l'approche de type "musique savante " adoptée par le Châtelet - n'est pas non plus expliqué. Le recours à des voix non travaillées aurait pourtant été possible, en plus d'être plus vraisemblable du point de vue de la reconstitution. Rappelons que les créatrices du Verfügbar aux Enfers étaient en majorité des chanteuses amateures qui reproduisaient probablement, à leur niveau, le style et la technique des grands interprètes de la chanson française d'avantguerre.

Le compositeur Christophe Maudot a justifié plusieurs de ses choix artistiques dans un court texte publié dans le dossier pédagogique du spectacle (Maudot 2007), mais aussi et surtout lors d'une intéressante conférence d'introduction à une représentation du Verfügbar aux Enfers donnée à Lorient en décembre $2010^{16}$. Maudot a ainsi expliqué que la composition du petit orchestre (deux violons, violoncelle, contrebasse, accordéon, flûte, clarinette, cor, trompette, percussions) résultait de sa volonté de trouver un équilibre entre l'instrumentation originale des sources musicales et celle qui aurait pu voir le jour à Ravensbrück. Ceci explique qu'il ait exclu certains instruments "de salon " (la harpe ou le piano) et qu'il ait retenu des instruments que les détenues ont pu voir dans les hangars du Bekleidung ${ }^{17}$, comme la clarinette et l'accordéon. Lors de sa conférence, il a exposé les grandes lignes de sa démarche

14 Document dactylographié sans référence émanant des services du Châtelet, Boîte "Verfügbar ", Chemise « Autour du Verfügbar aux Enfers (2005-2006) », Fonds Tillion, BnF.

15 Sur la grande variété de sources musicales convoquées dans Le Verfügbar aux Enfers, voir l'article de Marie-Hélène Benoit-Otis et Philippe Despoix dans le présent numéro.

16 Spectacle co-produit par ORPHÉE-Théâtre(s), le Grand Théâtre de Lorient et le Conservatoire de Lorient, et mis en scène par Jean-Michel Fournereau et Marc Sollogoub. Ce spectacle reprenait l'arrangement musical et les costumes de la production du Châtelet.

17 Les prisonnières affectées au Bekleidung devaient trier les vêtements et objets volés par les nazis et déposés dans de grandes halles. C'est ce lieu que Tillion choisit comme décor pour l'acte III du Verfügbar aux Enfers. 
de compositeur-arrangeur, basée sur le principe d'une fidélité aux sources musicales originales de l'opérette-revue. Lorsque le texte d'un numéro musical du Verfügbar aux Enfers est plus court que celui de la pièce ou chanson originale (par exemple dans le tango Il pleut sur la route à l'acte $\mathrm{II}^{18}$ ), il a pris le parti d'ajouter au texte de Tillion de nouvelles paroles afin de compléter le morceau. En revanche, quand le texte de Tillion est plus long que le texte original (c'est le cas notamment du duo de Ciboulette, dont la version proposée dans Le Verfügbar aux Enfers pose par ailleurs plusieurs problèmes de forme ${ }^{19}$ ), il a été décidé d'opérer des coupures afin de conserver la physionomie des morceaux d'avant-guerre repris par Tillion. Ce travail a été réalisé en collaboration avec la dramaturge Géraldine Keiflin, qui a également modifié plusieurs passages du texte parlé pour le rendre plus intelligible. Ce choix qui privilégie les sources musicales existantes rejoint la démarche plus générale de production d'une partition en bonne et due forme, même si celle-ci implique parfois de s'écarter du texte littéraire. Ainsi Le Verfügbar aux Enfers est-il ici transformé en " opérette ", la partie " revue " étant gommée, tout comme le suggère le sous-titre " une opérette à Ravensbrück » choisi par les éditions de La Martinière. On notera à ce sujet que le spectacle du Châtelet est lui-même sous-titré " Une opérette à Ravensbrück ", et que nombre de productions ultérieures ont également repris ce sous-titre de l'édition, remonté quelquefois en premier titre.

Le travail d'adaptation réalisé pour le Châtelet a également consisté à ajouter de nouvelles musiques. C'est le cas du « Prologue», court numéro purement instrumental ajouté en introduction, et dans lequel Maudot reprend un thème d'Orphée aux Enfers d'Offenbach (issu de la chanson d'Aristée de l'acte I et également présent dans l'ouverture de l'opéra-bouffe d'Offenbach). Autre exemple, dans le numéro suivant (qui ne contient pas de source musicale), Maudot insère au milieu de gammes ascendantes et descendantes le très célèbre thème de la "Habanera " de Carmen, d'une part pour éviter la monotonie (le texte est très long) et d'autre part parce qu'il lui semble intéressant de convoquer le personnage de Carmen, symbole de rébellion et de liberté, par le biais de cette référence musicale connue de tous ${ }^{20}$.

D'autres passages du Verfügbar aux Enfers obligent par ailleurs l'adaptateur à faire preuve d'invention, comme le moment de l'acte I faisant référence à $P h i$-Phi, une opérette de l'entre-deux-guerres ${ }^{21}$. Ici, pas de numéro chanté, mais simplement une référence musico-théâtrale à un air de l'opérette décrivant la poitrine féminine ("Les Petits Païens ») lorsqu'il est question des seins des détenues, qui, aux dires du naturaliste, « ne sont plus des saints, mais des martyrs » (Tillion 2005, p. 67 ; voir figure 1). Tillion indique en bas de page : «L'orchestre fredonne, bouche fermée, l'air

18 Pour plus de détails sur ce passage du Verfügbar aux Enfers, voir l'article de Marie-Hélène Benoit-Otis et Philippe Despoix dans le présent numéro.

19 Voir à ce sujet les articles de Christophe Gauthier et de Catherine Harrison-Boisvert et Caroline Marcoux-Gendron dans le présent numéro.

20 Les informations relatives aux intentions de Christophe Maudot sont tirées de la conférence qu'il a donnée au Grand Théâtre de Lorient le 4 décembre 2010.

21 Sur Phi-Phi, voir l'article de Pascal Blanchet dans le présent numéro. 
de Phi-Phi («Les Petits Païens ») mais sur un rythme de marche funèbre» (Tillion 2005, p. 67). Cette indication est particulièrement difficile à comprendre. Si l'« orchestre » semble en réalité renvoyer au chœur, comme dans la tragédie grecque, la mention $\mathrm{du}$ « rythme de marche funèbre » demeure bien mystérieuse. L'adaptateur doit donc trouver lui-même une solution qui fonctionne. Maudot choisit ici d'introduire la marche funèbre de Chopin (issue du troisième mouvement de la Sonate $n^{\circ} 2$ op. 35 pour piano), sans doute la marche funèbre la plus connue du grand public, mais il ne se contente pas d'en citer le rythme : il en reprend tous les paramètres dans un arrangement pour chœur et orchestre entendu après une adaptation de la chanson originale des « Petits Païens » chantée par le Naturaliste.

Extrait vidéo 1 : David Unger, Le Verfügbar aux Enfers. Une opérette à Ravensbrück, Théâtre du Châtelet (2007), Extrait de l'acte I, "Les Petits Païens ». Écouter.

Maudot ne fond donc pas les deux références (chanson d'opérette et rythme de marche funèbre) de façon simultanée, mais les cite l'une après l'autre, tout en insistant sur la référence à la marche funèbre. Il termine le numéro par un passage dans un style grégorien (chœur a cappella à l'unisson) sur l'incipit du psaume « De profundis clamavi " cité dans le texte de Tillion en référence à la sainteté des seins devenus martyrs. Si l'on peut émettre quelques réserves sur l'adaptation musicale de ce passage qui ne respecte pas vraiment la " consigne » donnée par Tillion ni même l'ordre des références musicales, l'effet est réussi dans la mesure où le public peut saisir l'humour résultant de la superposition des références et des registres.

Le choix de mise en scène de ce numéro est en revanche plus contestable : tandis qu'il chante l'air coquin des «Petits Païens » avec une ironie grinçante, le naturaliste fait défiler les détenues qui lui montrent une à une leur poitrine. Il les repousse alors avec violence, tirant même les cheveux de l'une d'entre elle. Cette rude inspection du corps des prisonnières par un naturaliste qui s'apparente ici à un bourreau confère une brutalité et un cynisme inexpliqués à cette référence aux " Petits Païens » avant tout caractérisée par la légèreté et l'autodérision. Ce passage témoigne également de l'atmosphère plutôt sombre de la mise en scène du Châtelet, ainsi que de la définition ambiguë du personnage du naturaliste qui oscille entre le meneur de revue, le conférencier, le bourreau et la victime (plus loin, le naturaliste se retrouve en corset et souffre d'intenses maux de ventre, à l'instar des détenues).

Une autre caractéristique importante de la production réside dans le choix d'intégrer de jeunes choristes et danseurs issus d'établissements scolaires et musicaux (collèges, conservatoires et maîtrise de Paris), comme en témoigne le document du Châtelet cité plus haut. Mêler les corps et les voix de jeunes amateurs à ceux d'artistes professionnels permet ici avant tout de faire découvrir la pièce à de nombreux adolescents, et potentiellement à leurs proches. L'enjeu est en effet à la fois pédagogique et mémoriel, car à travers l'étude et l'interprétation de ce texte très puissant et porteur, il s'agit de transmettre aux jeunes les valeurs de la Résistance et la mémoire de la déportation, l'objectif sous-jacent étant que les nouvelles générations les transmettent à leur tour aux générations futures. L'idée d'un hommage intergénérationnel aux résistants et aux déportés est très nette lorsque des rescapés des camps assistent aux représentations - on pense en particulier à celle qui s'est tenue sur les lieux mêmes du camp 
de Ravensbrück en avril 2010 à l'occasion du 65 anniversaire de sa libération ${ }^{22}$. Le silence toutes lumières éteintes qui conclut les représentations du Châtelet, avant que ne descende des cintres un grand portrait de Tillion, relève également de cet hommage, centré cette fois sur la personnalité de la principale auteure de l'opérette-revue. Signalons enfin que ces deux premières représentations complètes de l'œuvre données au Châtelet les 2 et 3 juin 2007 soulignent (presque jour pour jour) le centième anniversaire de la naissance de Tillion (1907-2008). «Pour les cent ans de Germaine Tillion, on ne pouvait rêver plus bel hommage que la création du Verfügbar aux Enfers par de jeunes artistes ", peut-on lire au verso du flyer ; le recto reprend l'affiche du spectacle ornée d'un récent portrait de l'ethnologue - un cliché qui renvoie lui aussi à la volonté de célébrer l'auteure de l'opérette-revue.

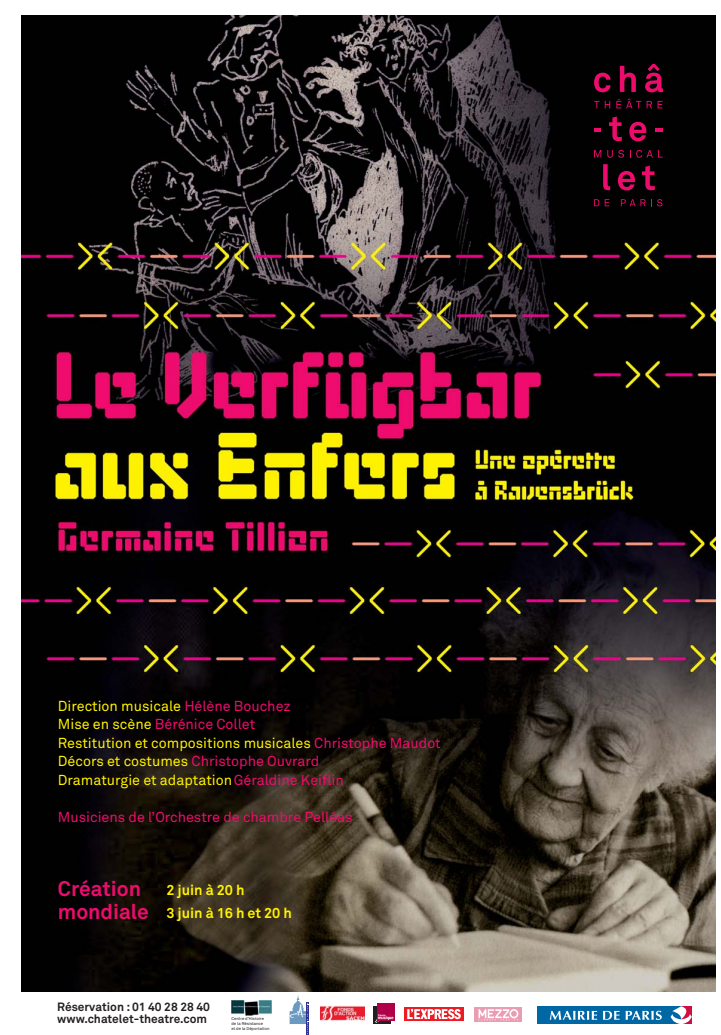

Figure 3 : Affiche du spectacle Le Verfügbar aux Enfers. Une opérette à Ravensbrück créé au Théâtre du Châtelet (2007). (C) Théâtre du Châtelet / typo Philippe Apeloig. Photo de Germaine Tillion : Eric Bergeron; dessin de France Audoul. La reproduction du dessin de France Audoul, les barbelés stylisés et le fond noir soulignent le contexte de création de l'œuvre, tandis que la photographie de Germaine Tillion en train d'écrire met l'accent sur l'hommage rendu à l'ethnologue et sur l'écriture comme acte de résistance.

22 Coproduit par le Théâtre du Châtelet et le Mémorial de Ravensbrück, ce spectacle reprenait la version du Châtelet mais dans une forme oratorio et moyennant de nouveaux arrangements. Il faisait intervenir de jeunes musiciens français (collégiens et lycéens parisiens) et allemands (Chœur de jeunes filles de Berlin et Musiciens de la Junge Philarmonie Brandenburg). 
Cette première version fait aujourd'hui autorité ${ }^{23}$ et a constitué un modèle pour une bonne partie des productions ultérieures. Plusieurs d'entre elles ont en effet repris l'adaptation théâtrale et musicale de Keiflin et Maudot : c'est le cas des spectacles montés à Seynod ${ }^{24}$ en 2009 , à Lorient ${ }^{25}$ et à Ravensbrück ${ }^{26}$ en 2010 , et à Nantes ${ }^{27}$ en 2011 - pour cette dernière production, le metteur en scène proposait toutefois une nouvelle adaptation du texte. Toutes ces représentations ont fait intervenir des jeunes collégiens et lycéens, musiciens appartenant à un ensemble ou bien élèves en école de musique ou en conservatoire pour constituer le chœur féminin et/ou l'ensemble instrumental. Cette approche pédagogique du Verfügbar aux Enfers trouve un prolongement dans un récent projet de l'Académie musicale de Villecroze. Spécialisée dans le soutien à la création et à la pratique musicale chez les jeunes, celle-ci a en effet commandé à Maudot une version chorale de l'œuvre, puis a organisé un stage à l'automne 2014 pour former les enseignants d'éducation musicale du secondaire à cette partition ${ }^{28}$. En mai 2015, des lycéens de région parisienne ont interprété cette nouvelle version chorale à l'occasion de l'entrée de Tillion au Panthéon ${ }^{29}$.

\section{De jeunes amateurs en quête de nouvelles sources musicales}

Une production se démarque toutefois de l'ensemble des spectacles incluant des jeunes interprètes et présentés à la suite des représentations du Châtelet. Il s'agit du spectacle réalisé par la troupe du Manoir de Saint-Avé ${ }^{30}$ (Bretagne) en 2008, une représentation faisant uniquement intervenir des jeunes de lycée et qui mobilisait des sources musicales totalement étrangères à l'opérette-revue. L'un des deux metteurs en scène s'explique : "pour montrer l'intemporalité de la pièce [...] nous avons

23 C'est cette production que l'Association Germaine Tillion choisit de montrer dans son récent film documentaire, Germaine Tillion par elle-même (2015), lorsqu'est évoquée l'opérette-revue.

24 Coproduction Centre de Pratique musicale d'Annecy, École de musique de Seynod, Auditorium Scène régionale de Seynod, avec la collaboration artistique du groupe Décembre et la mise en scène de Christian Taponard.

25 Voir la note 16.

26 Voir la note 22.

27 Coproduction Théâtre de l'Entracte, Hop'La Copro. En partenariat avec le Théâtre de l'Entracte, Angers Nantes Opéra, l'Ensemble Utopik et l'Université Permanente de Nantes, avec la mise en scène et l'adaptation d'Henri Mariel.

28 Projet «Création de matériel pédagogique autour d'une opérette écrite à Ravensbrück » réalisé par l'Académie musicale de Villecroze, août 2014. Voir la présentation et le document d'accompagnement disponibles en ligne, http://education-musicale.discip.ac-caen.fr/spip.php?article84 (consulté le

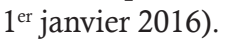

29 Les chœurs des lycées Albert Schweitzer du Raincy et Guillaume Apollinaire de Thiais se sont produits au Conservatoire à rayonnement régional de Paris et au Centre culturel du Raincy les 28 et 29 mai 2015.

30 Pièce « interprétée par le groupe d'Ados de la troupe du Manoir de Saint-Avé, mise en scène par Sophie De Buisson et Jacky Kerneur ». Voir http://troupedumanoir.fr/le-verfugbar-aux-enfers/ (consulté le $1^{\text {er }}$ janvier 2016). 
adapté sur des musiques contemporaines les paroles des chansons de l'époque de madame Tillion [...] dans le but que les jeunes adhèrent au projet et cherchent les airs les mieux adaptés aux paroles ${ }^{31}$ ". Ce choix de faire appel à des musiques récentes permet de contourner la difficulté qui se pose pour toute mise en scène contemporaine du Verfügbar aux Enfers : comment faire saisir au public d'aujourd'hui l'effet humoristique né du détournement de sources musicales désormais oubliées ? Si le parti pris de la troupe du Manoir de Saint-Avé présente le grand intérêt de faire appel à la mémoire collective des interprètes - comme ce fut le cas pour les détenues de Ravensbrück - et du public d'aujourd'hui, il n'est pas sans poser problème. Tout d'abord, le fait de calquer les textes de la pièce sur de nouvelles chansons génère un inévitable problème de prosodie : la scansion rythmique des chansons d'origine ne peut en effet être respectée. De plus, en effaçant les sources musicales de l'opérette-revue, cette démarche induit une compréhension partielle de l'œuvre, puisque les textes « musiqués » du Verfügbar aux Enfers sont consubstantiellement liés à toutes les dimensions musicales (rythme, mélodie, phrasé, vocalité, forme, etc.) et psychologiques (registre amoureux, comique, etc.) de leurs références d'origine. Enfin, dans le cas présent, le choix de certaines chansons (qui appartiennent davantage à la mémoire collective des parents d'adolescents de 2010 qu'aux adolescents eux-mêmes, ou bien qui se situent dans un registre ou un imaginaire trop éloigné de celui de la pièce ${ }^{32}$ ) pose problème. Malgré ces réserves, cette production a le grand mérite de proposer une lecture originale et une véritable re-création de l'œuvre par de jeunes amateurs, et donc de poursuivre la première démarche des créatrices de l'opérette-revue.

\section{Le duo du Théâtre de la Petite Montagne}

La dimension pédagogique est également présente dans un spectacle d'une tout autre nature, celui du Théâtre de la Petite Montagne, d'abord représenté dans le Jura en 2010 avant d'être transporté à Paris (dans la crypte de l'Église Saint-Sulpice) et dans de nombreuses villes de province où il est encore présenté en 2016. Cette production se distingue à la fois par une grande force théâtrale et une grande économie de moyens. Deux comédiennes seulement se partagent les dialogues et chansons ( a cappella) en faisant évoluer d'étranges marionnettes dans un espace scénique très réduit et des décors et lumières minimalistes.

31 Courriel de Jacky Kerneur à l'auteure, 13 octobre 2014.

32 On pense par exemple aux chansons « Les Copains d'abord » de Georges Brassens et « Ouragan » de Stéphanie de Monaco. 


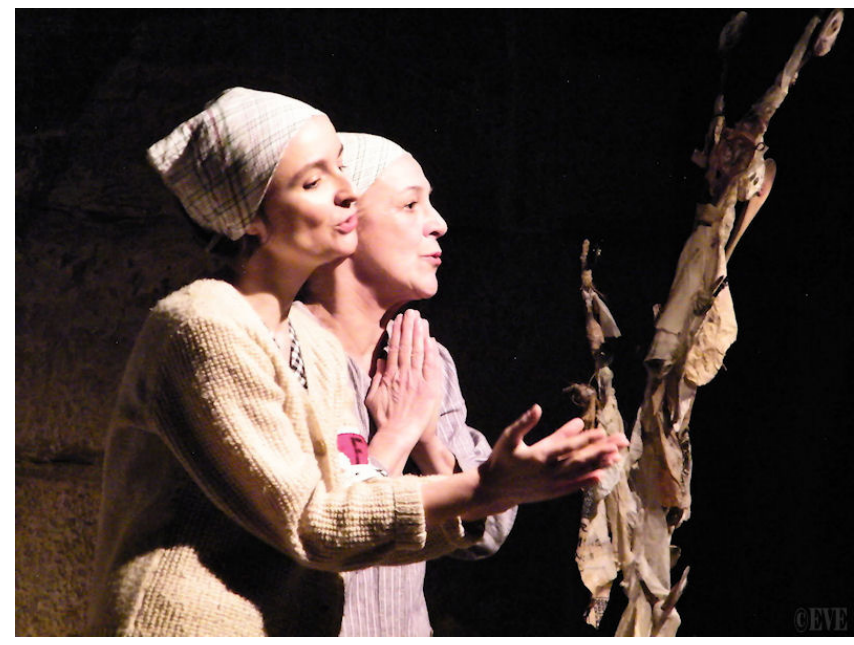

Figure 4: Photographie de scène du spectacle Une opérette à Ravensbrück (2013), Théâtre de la Petite Montagne, Paris, Église Saint-Sulpice.

(C) Théâtre de la Petite Montagne. Photo : Eve Thiel.

Ce «spectacle de mémoire, à but pédagogique » (Une opérette à Ravensbrück [2014], p. $\left.9^{33}\right)$, proposé à tout public et dans différents lieux y compris des établissements scolaires, est précédé d'une introduction qui permet au spectateur " de mieux appréhender la construction de cet écrit, entrer dans ses subtilités, sa réalité parfois crue et souvent déconcertante ", et suivi d'un débat (avec la participation éventuelle d'anciens déportés ou résistants) « dont le message principal est le devoir de mémoire et de conscience " (ibid., p. 10).

Le Théâtre de la Petite Montagne propose une lecture très personnelle de l'opérette-revue, qui apparaît davantage comme une œuvre de théâtre librement interprétée que comme une œuvre musico-théâtrale. Si le texte prime, il n'y a pas ici de recherche d'une fidélité - même partielle - à l'œuvre, mais plutôt une prise en compte très fine $\mathrm{du}$ contexte dans certains choix artistiques. Ainsi les marionnettes qui figurent les différents personnages de l'opérette sont-elles conçues «à partir de presque rien " pour signifier le dénuement matériel des détenues. "Les femmes qui étaient internées en camp de concentration ne possédaient rien, elles n'avaient plus rien à elles ", explique Hélène Saïd, la créatrice des marionnettes.

Par conséquent un petit morceau de ficelle, un bouton de chemise trouvé dans la boue du camp devenaient précieux. C'est de cette manière-là que j'ai voulu travailler sur les marionnettes de l'opérette. Tenter de créer à partir de presque rien, s'étonner que la beauté puisse encore surgir d'un assemblage de bouts de bois, d'écorces, de papiers, de chiffons (ibid.).

Plus généralement, le fait que l'opérette ait été écrite dans un camp est à l'origine du choix d'une mise en scène particulièrement épurée, qui vise non pas à représenter le 
camp, mais à le suggérer. «Que l'univers concentrationnaire soit juste suggéré le rend d'autant plus présent ", expliquent les créateurs (Une opérette à Ravensbrück [2014], p. 9). L'effet est réussi, " avec pudeur et surtout sans faux réalisme ", comme le note François Marcot, historien et président des Amis du Musée de la Résistance et de la Déportation (dans ibid., p. 13).

De même, le choix de proposer des voix nues et non travaillées semble témoigner d'un attachement particulier au contexte de création de l'œuvre qui, rappelons-le, fut conçue par des femmes qui n'étaient pas des chanteuses de métier. De fait, les deux excellentes comédiennes du Théâtre de la petite montagne ne sont pas chanteuses professionnelles ; si elles chantent avec application, on peut être gêné par plusieurs imperfections dans l'exécution vocale. Leur interprétation qui privilégie l'intention ainsi que la clarté de la diction n'en est pas moins convaincante. On pourra le constater en visionnant un extrait du premier acte du spectacle contenant le passage où le naturaliste décrit l'espèce du Verfügbar suivi de la chanson « On m'a dit : il faut résister » (acte $\mathrm{I})^{34}$ interprétée à nu et à l'unisson.

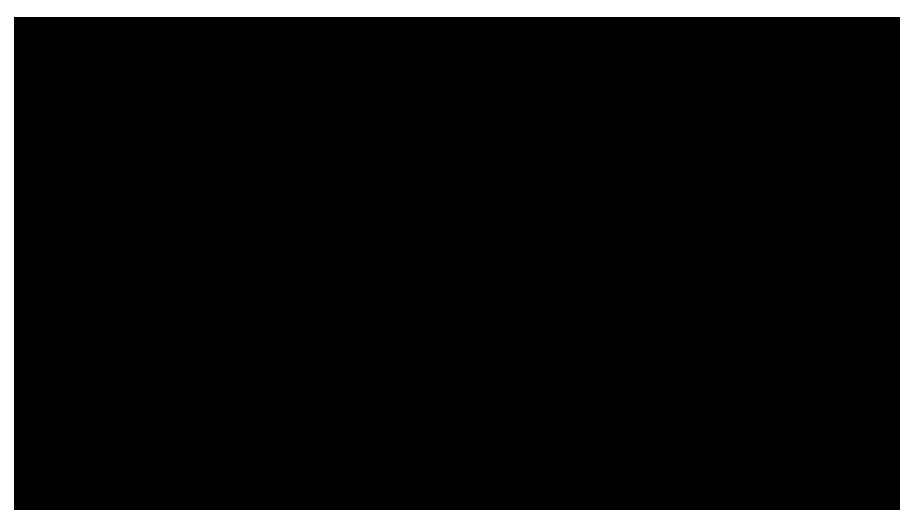

Extrait vidéo 2 : Annie Vacelet-Vuitton, Une opérette à Ravensbrück (2010), Théâtre de la Petite Montagne, Paris, Église Saint-Sulpice. Extrait de l'acte I, "On m'a dit il faut résister». () Théâtre de la Petite Montagne.

\section{Au chour du texte : la production Femmes et Résistances}

Une autre production récente se distingue par sa grande qualité théâtrale - ce sera notre dernier exemple. Il s'agit d'un spectacle marseillais issu de la société en participation Femmes et Résistances (regroupant les compagnies Trafic d'art II, Nuits Blanches en Compagnie et Léda Atomica Musique) et réalisé en coproduction avec le Théâtre Gyptis de Marseille où il a été créé en 2011. La force de ce spectacle réunissant neuf comédiennes et comédiennes-musiciennes réside selon nous dans la juste interprétation du texte, mais aussi dans la grande inventivité de l'adaptation proposée par Danielle Stéfan (mise en scène et adaptation), Hélène Arnaud (adaptation) et Alain Aubin (composition et arrangements musicaux).

34 Sur cet air, voir l'article de Catherine Harrison-Boisvert et Caroline Marcoux-Gendron dans le présent numéro. 
On est d'abord frappé par la compréhension des processus de création de l'œuvre, qui se manifeste notamment par les décors, tel le tableau noir ornant le fond de la scène de l'acte I et que les comédiennes remplissent peu à peu de leurs mots et dessins à la craie. Ce décor de salle de classe progressivement investi par les personnages offre un cadre tout à fait approprié à la conférence du naturaliste tout en proposant une manifestation concrète de l'écriture collective et de la trace. On notera également que le naturaliste est ici joué par une femme, ce qui suggère une mise en abîme de la représentation théâtrale : le spectateur peut ainsi voir dans ce personnage celui d'une détenue jouant elle-même ce rôle du conférencier-meneur de revue. C'est donc à une sorte de création différée de cette pièce conçue au sein d'un environnement uniquement féminin que le public est ici convié. De même, la forte présence du chant a cappella, ou discrètement accompagné par un ou deux instruments joués sur scène (accordéon et violon - qui jouent ponctuellement le rôle d'instrument de remémoration ${ }^{35}$ ) rappelle clairement l'univers dans lequel l'œuvre est née. L'accompagnement peut quelquefois n'être qu'un discret soutien, comme dans « Chanson triste " (acte I) exécutée à pleine voix et presque a cappella par une des comédiennes-chanteuses.

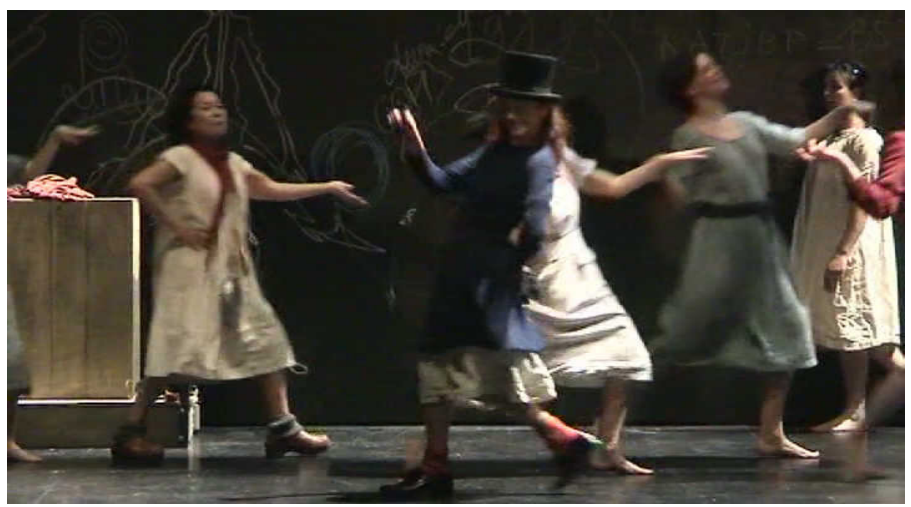

Extrait vidéo 3 : Frédéric Vidal, Une opérette à Ravensbrück. Le Verfügbar aux Enfers (2012), production Aime le maudit, Marseille, Théâtre Gyptis. Extrait de l'acte I, "Chanson triste». (C) Femmes et Résistance.

Cette adaptation révèle la puissance lyrique de la mélodie de Duparc, mais en la libérant de sa trame rythmique (le flot de doubles croches de l'accompagnement original est ici supprimé) et en lui adjoignant d'étranges couleurs produites par les lignes de violon et d'accordéon. Ce choix d'interprétation prend une signification toute particulière lorsqu'on sait que ce numéro a vraisemblablement été " amené " et chanté à Tillion par une codétenue, l'artiste lyrique française Jany Silvaire. Cette dernière se souvient en effet avoir chanté cette mélodie à sa sœur malade au camp

35 Dans le premier numéro musical ("J'avais une grande maison », composition d'Alain Aubin), le déplacement de l'accordéoniste qui entame le morceau en s'approchant de Nénette avant que cette dernière se mette à chanter peut être vu comme une manifestation visible du processus de remémoration musicale des créatrices de l'opérette. 
de Neubrandenburg où elle a été déportée après sa détention à Ravensbrück ${ }^{36}$. Faire chanter aujourd'hui cette prière d'espoir par une voix lyrique presque à nu et s'épanouissant librement n'est donc pas seulement une solution originale et artistiquement convaincante ; ce choix fait aussi directement écho à la genèse de l'opérette-revue.

Cette attention portée au processus de création de l'opérette-revue se retrouve dans la recherche attentive de ses sources musicales, recherche qu'avait aussi menée l'équipe du Châtelet ${ }^{37}$. La pièce a également été enrichie de quelques nouvelles musiques. Aux 27 numéros chantés et à la pièce instrumentale ${ }^{38}$ cités dans le texte, les adaptateurs ont ajouté une composition d'Alain Aubin (La Valse des morts de faim), ainsi que le célèbre galop d'Orphée aux Enfers d'Offenbach. Le galop est présenté à deux reprises dans une version chorale a cappella particulièrement fantaisiste, qui met 1'accent sur l'humour et l'autodérision des détenues. Le morceau, dont les parties instrumentales et vocales sont chantées sur le son " pa », est d'abord interprété par les comédiennes pendant la déclamation du Prologue, mettant ainsi en valeur l'hommage à Offenbach et à l'opérette. Il est repris lorsque Nénette partage un morceau de pain avec ses compagnes (acte I, p. 26) : après la distribution, les personnages entonnent progressivement le galop sur des « pa » s'apparentant à des cris de basse-cour puis entament une brève chorégraphie d'animaux de poulailler surexcités.

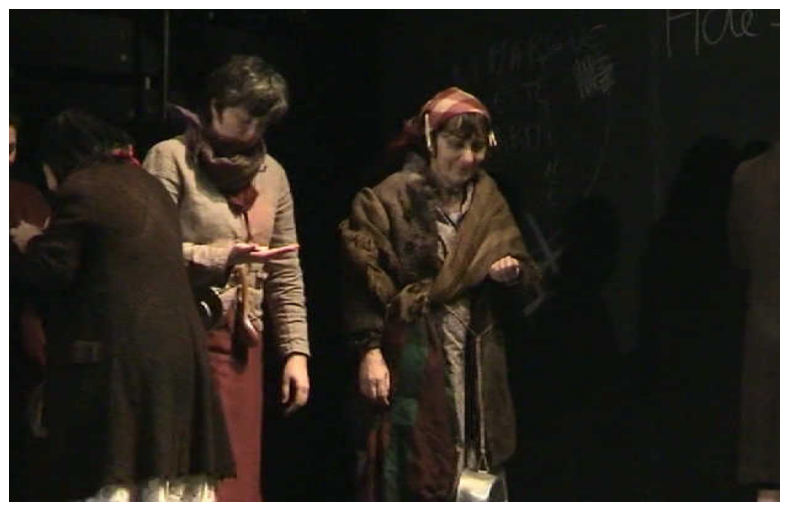

Extrait vidéo 4 : Frédéric Vidal, Une opérette à Ravensbrück. Le Verfügbar aux Enfers (2012), production Aime le maudit, Marseille, Théâtre Gyptis.

Extrait de l'acte I. () Femmes et Résistance.

S'il ne figure pas dans le texte du Verfügbar aux Enfers, ce « cancan des poules » rejoint tout à fait l'un des ressorts principaux de l'opérette-revue : la vitalité et le caractère exutoire du rire collectif, qui permet de lutter contre l'horreur et la coercition.

36 Jany Silvaire évoque ce souvenir dans le premier de deux entretiens de remémoration réalisés l'un chez Germaine Tillion à Saint-Mandé le 20 avril 2005, et l'autre chez Françoise Brustlein le 28 avril 2005. Les enregistrements de ces entretiens sont conservés par l'Association Germaine Tillion.

37 Notons que les créateurs marseillais ont découvert deux nouvelles sources musicales : « Elle est en peau d'vache » (acte I) et "Une canne et des gants » (acte I).

38 La Danse macabre, op. 40 de Camille Saint-Saëns. 
De façon générale, les choix artistiques suivent de très près la forme et le sens de l'œuvre, mettant l'accent par exemple sur la revue, sur le registre tragicomique, ou encore sur la " choralité du texte » (Arnaud et Stéfan 2011, p. 4), particulièrement valorisée dans ce spectacle. Cette choralité n'est pas seulement perceptible dans les moments spécifiquement dédiés aux chœurs, elle apparaît également dans certains passages que les créateurs du spectacle ont décidé de " mutualiser » en y intégrant les autres personnages constitutifs du chœur - réduit à neuf individualités dans le cas de ce spectacle. On pense par exemple au duo de Ciboulette (acte II), "Nous avons fait un beau voyage ", qui devient une polyphonie alternée entre les neuf comédiennes rassemblées autour d'un drap faisant office de nappe puis de serviette de pique-nique. Le détournement du duo de Reynaldo Hahn en voyage gastronomique imaginaire et collectif est ici particulièrement bien rendu, tout comme le dialogue qui le précède (la constitution collective du menu du jour), ponctué de cris de plaisir des personnages. Dans ce moment dialogué puis chanté surgit toute l'énergie créatrice du groupe de détenues, et l'adaptation proposée par Femmes et Résistances met précisément en valeur l'invention collective des prisonnières échafaudant ici une double évasion imaginaire, à la fois géographique et gastronomique, un "beau voyage " dans " tous les jolis coins de France » permettant in fine le retour tant espéré dans la patrie.

\section{CONCLUSION}

Depuis sa publication, Le Verfügbar aux Enfers a fait 1'objet de brèves études de nature historique ou historiographique - et dans une moindre mesure littéraire et esthétique - et a donné lieu à de nombreuses productions musico-théâtrales, dont la première et la plus connue est celle réalisée au Théâtre du Châtelet en 2007. L'opérette-revue continue aujourd'hui d'être régulièrement représentée en France et a même été donnée récemment aux États-Unis dans une traduction anglaise ${ }^{39}$. Si une étude approfondie des mises en scènes contemporaines de l'œuvre reste à faire, les quelques coups de sonde proposés ici auront mis en évidence plusieurs grandes tendances dans les reconstitutions actuelles. Nous avons vu que les artisans de la production du Châtelet ont traité Le Verfügbar aux Enfers comme une composition musicale traditionnelle en procédant au transfert du manuscrit de Tillion vers une partition avec tous les arrangements nécessaires pour faire coller le texte aux musiques d'emprunt. À cette approche musicale « savante » de l'œuvre s'est ajoutée une dimension pédagogique et mémorielle, marquée notamment par le souci de faire intervenir sur scène de jeunes musiciens. Plusieurs productions ont ensuite repris les principes adoptés par cette première adaptation. Nous avons pu observer par ailleurs des mises en scène plus théâtrales et davantage centrées sur la forme et le sens du texte. D'autres exemples ont également montré la volonté de suggérer l'univers dans lequel l'œuvre est née, volonté qui a quelquefois suscité l'intégration de nouveaux matériaux.

39 In the Underworld. A Darkly Comic Operetta, University of South Maine, 18-27 avril 2014. Mise en scène de Meghan Brodie, traduction d'Annie et Karl Bortnick, arrangement et composition de Christophe Maudot. 
Il n'existe certes pas une seule option satisfaisante, d'autant plus que ce texte écrit en déportation n'est pas une œuvre musicale ou théâtrale au sens usuel du terme. Le jugement habituel sur les mises en scène lyriques se focalise sur la légitimité d'une interprétation en tant que traduction d'un texte et restitution de l'univers d'où l'œuvre est née (Nattiez 2007, p. 209). Si une production d'opéra ne peut donner lieu à une fidélité intégrale, la notion même de fidélité - fût-elle partielle ou locale - pose ici problème, puisqu'on ne dispose pas de partition, qu'il n'existe pas de mise en scène originale et qu'il est impossible de recréer l'univers dans lequel l'œuvre a vu le jour. Les adaptateurs du Verfügbar aux Enfers doivent donc chercher ailleurs pour restituer la grande force de ce texte musico-théâtral, œuvre de résistance mais aussi d'une très haute tenue littéraire et artistique.

\section{BIBLIOGRAPHIE}

Andrieu, Claire (2015), "Le Verfügbar aux Enfers. Une lecture historique ", dans Les armes de l'esprit, Germaine Tillion 1939-1954, catalogue de l'exposition présentée par le Musée de la Résistance et de la Déportation de Besançon du 26 mai au 20 septembre 2015, Citadelle de Besançon, Besançon, Musée de la Résistance et de la Déportation, p. 71-77.

Arnaud, Hélène, et Danielle Stéfan (2011), "Présentation ", dans Une opérette à Ravensbrück. Le Verfügbar aux Enfers de Germaine Tillion, une production Femmes et Résistances, mise en scène Danielle Stéfan, dossier spectacle.

Association Germaine Tillion (2015), Germaine Tillion par elle-même, documentaire, Paris, 65 min.

Audhuy, Claire (2013), "Le théâtre dans les camps nazis. Réalités, enjeux et postérité ", thèse de doctorat, Université de Strasbourg.

Audhuy, Claire (2014), «Du langage des hommes "normaux" », Horizons/Théâtre, nº 3, p. 3-15.

Brodziak, Sylvie (2013), «Rires de femmes à Ravensbrück. Le Verfügbar aux Enfers de Germaine Tillion », dans Violaine Houdart-Merot (dir.), Rires en francophonie, Amiens, Encrage Université, p. 135154.

Font, Emmanuel (2015), «Théâtre et déportation. Le rire de Germaine Tillion face à l'expérience concentrationnaire ", dans Les armes de l'esprit, Germaine Tillion 1939-1954, catalogue de l'exposition présentée par le Musée de la Résistance et de la Déportation de Besançon du 26 mai au 20 septembre 2015, Citadelle de Besançon, Besançon, Musée de la Résistance et de la Déportation, p. 89-95.

Gilzmer, Mechtild (2013), " De Blanche-Neige à Rieucros au Verfügbar de Ravensbrück. Pour une mémoire transnationale des camps pour femmes dans les années 40 ", dans Armelle Mabon et Gwendal Simon (dir.), L'engagement à travers la vie de Germaine Tillion, Paris, Riveneuve éditions Bretagne, p. 145-157.

Giner, Bruno (2011), Survivre et mourir en musique dans les camps nazis, Paris, Berg.

Losco-Lena, Mireille (2011), "Rien n'est plus drôle que le malheur». Du comique et de la douleur dans les écritures dramatiques contemporaines, Rennes, Presses universitaires de Rennes.

Loselle, Andrea (2010), " Performing in the Holocaust. From Camp Songs to the Song Plays of Germaine Tillion and Charlotte Salomon ", The Space Between, vol. 6, n 1, p. 13-38.

Maudot, Christophe (2007), "Le Verfügbar aux Enfers. Une opérette-revue à Ravensbrück. Quelques remarques pour une restitution-création ", dans Le Verfügbar aux Enfers. Une opérette à Ravensbrück, dossier pédagogique, Châtelet, Théâtre musical de Paris, p. 40-41. 
Maudot, Christophe (2015), "Les soirées d'une étudiante en ethnologie à Paris (1926-1934) et l'opérette-revue Le Verfügbar aux Enfers (Ravensbrück, octobre 1944) ", dans Les armes de l'esprit, Germaine Tillion 1939-1954, catalogue de l'exposition présentée par le Musée de la Résistance et de la Déportation de Besançon du 26 mai au 20 septembre 2015, Citadelle de Besançon, Besançon, Musée de la Résistance et de la Déportation, p. 79-86.

Musée de la Résistance et de la Déportation de Besançon (2015), Les armes de l'esprit, Germaine Tillion 1939-1954, catalogue de l'exposition présentée par le Musée de la Résistance et de la Déportation de Besançon du 26 mai au 20 septembre 2015, Citadelle de Besançon, Besançon, Musée de la Résistance et de la Déportation.

Nattiez, Jean-Jacques (2007), " Mise en scène lyrique, interprétation et sémiologie ", dans Laetitia Petit (dir.), Prétentaine, "Opéra. Mises en scènes et représentations théâtrales ", n $20 / 21$ (mars), p. 205-257.

Reid, Donald (2007a), « Available in Hell. Germaine Tillion's Operetta of Resistance at Ravensbrück », French Politics, Culture \& Society, vol. 25, n 2, p. 141-149.

Reid, Donald (2007b), Germaine Tillion, Lucie Aubrac, and the Politics of Memories of the French Resistance, Cambridge, Cambridge Scholars Publishing.

Salomon, Charlotte (2015), Vie ? ou Théâtre ?, traduit par Anne-Hélène Hoog et Michel Roubinet, Paris, Le Tripode.

Steinberg, Michael P., et Monica Bohm-Duchen (dir.) (2006), Reading Charlotte Salomon, Ithaca, Cornell University Press.

Tillion, Germaine (1988), Ravensbrück, Paris, Seuil.

Tillion, Germaine (2005), Le Verfügbar aux Enfers, une opérette à Ravensbrück, présentation de Tzvetan Todorov et Claire Andrieu, Paris, La Martinière.

Toulouse-Lautrec, Béatrix de (1981), La victoire en pleurant, Paris, France Empire.

Une opérette à Ravensbrück ([2014]), dossier spectacle téléchargeable sur le site du Théâtre de la Petite Montagne, http://www.theatre-biolopin.com/une-operette-a-ravensbruck.htm, consulté le $1^{\text {er }}$ janvier 2016.

Voolen, Edward van (dir.) (2005), Charlotte Salomon, Vie ? ou Théâtre ?, Paris, Prestel/Musée d'art et d'histoire du Judaïsme.

Vidal, Frédéric (2012), Une opérette à Ravensbrück. Le Verfügbar aux Enfers, court métrage documentaire, production Aime le mot dit, Marseille, Théâtre Gyptis, 56 min. 